\title{
M icroestructura y propiedades ópticas de películas de bismuto y óxido de bismuto depositadas con magnetrón desbalanceado
}

\author{
Diana M aría Otálora', G iovany O rozco², J hon J airo O laya-F lórez²,* \\ ${ }^{1}$ Departamento de Física, Universidad Nacional de Colombia, Bogotá, Colombia \\ ${ }^{2}$ Departamento de Ingeniería Mecánica y Mecatrónica, Universidad Nacional de Colombia, Bogotá, Colombia
}

\begin{abstract}
Resumen
El bismuto y el óxido de bismuto son materiales de gran interés tecnológico y teórico debido a sus amplias propiedades ópticas y eléctricas. En este trabajo, se fabricaron por medio de la técnica de magnetrón desbalanceado (UBM) películas delgadas de bismuto $(\mathrm{Bi})$ y óxido de bismuto $\left(\mathrm{Bi}_{2} \mathrm{O}_{3}\right)$ a temperatura ambiente y sobre sustratos de vidrio, con el objeto de evaluar sus propiedades con respecto al método de crecimiento. Las propiedades microestructurales de las muestras se estudiaron mediante difracción de rayos X ( $X$-ray diffraction, XRD) y microscopía láser confocal (confocal laser scanning microscopy, CLM), en tanto que su composición elemental se analizó con la técnica de espectroscopia de electrones Auger (Auger electron spectroscopy, AES) y sus propiedades ópticas con el método de la espectroscopia ultravioleta visible (UV/Vis) en el rango de longitudes de onda de $500-1800 \mathrm{~nm}$. Con base en los resultados de XRD se observó que ambos materiales son policristalinos, con una estructura romboédrica para el bismuto y la fase metaestable para el óxido de bismuto. Mediante el análisis de las propiedades ópticas se obtuvieron valores en la banda de energía prohibida de 2,3 eV y 2,27 eV para el bismuto y el óxido de bismuto, respectivamente.
\end{abstract}

Palabras clave: bismuto, microestructura, óxido de bismuto, propiedades ópticas, magnetrón desbalanceado.

Microstructure and optical properties of bismuth and oxide bismuth films using unbalanced magnetron

\begin{abstract}
A bstract
Bismuth and bismuth oxide are materials of technological and theoretical interest because of their optical and electrical properties. Thin films of bismuth (Bi) and bismuth oxide (Bi2O3) on glass substrates were produced at room temperature using the unbalanced magnetron sputtering (UBM) technique. The microstructural characterization of samples was studied using x-ray diffraction (XRD) and confocal laser microscopy (CLM), while their elemental composition was analyzed with Auger electron spectroscopy (AES) and their optical properties with ultravioletvisible (UV/Vis) spectroscopy in the wavelength range of $500-1800 \mathrm{~nm}$. XRD results showed that both materials have a polycrystalline character, with a rhombohedral structure for bismuth and phase for bismuth oxide. The energy band gap was $2.3 \mathrm{eV}$ and $2.27 \mathrm{eV}$ for bismuth and bismuth oxide, respectively.
\end{abstract}

Key words: Bismuth, microstructure, bismuth oxide optical properties, unbalanced magnetron sputtering.

\section{Introducción}

El semimetal bismuto es uno de los pocos metales que se expanden al solidificarse; tiene una conductividad térmica menor que la de cualquier otro metal, exceptuando el mercurio; es inerte en el aire seco a temperatura ambiente, pero en un ambiente húmedo se oxida con facilidad, y por encima de su punto de fusión forma rápidamente películas de óxido (Yang, 2008). Cuando el bismuto se crece en la forma de una película delgada exhibe un comportamiento inusual comparado con las propiedades del material en volumen. Un ejemplo de ello se observa en la tendencia de la resistividad versus la disminución de la temperatura, lo que está relacionado con el espesor de la película que satura a bajas temperaturas. El bismuto presenta características particulares, tales como la marcada diferencia en sus propiedades de superficie y volumen, la banda de energía prohibida, la existencia de estados electrónicos superficiales que se cruzan con el nivel de Fermi, una alta movilidad de portadores, su baja masa efectiva, su baja densidad de portadores y un gran camino libre medio, entre otras (Depablos, 2013; H ofmann, 2006). El bismuto es uno de los elementos más usados para aplicaciones en escalas nanométricas como las

\footnotetext{
*Correspondencia:

Jhon Jairo Olaya-Flórez, jjolaya@unal.edu.co

Recibido: 23 de septiembre de 2014

A ceptado: 28 de enero de 2015
} 
películas delgadas, las nanopartículas, o las multicapas nanoestructuradas (L utskii, 1965; Sandomirskii, 1967; Dresselhaus, et. al., 2003; Hicks \& Dresselhaus, 1993; Lin, et al., 2000; Iyengar, et al., 2012; Boffoué, 1998), y puesto que es un metal pesado no contaminante, existe gran interés por desarrollarlo como reemplazo de otros metales pesados, por ejemplo, en electrodos para la detección de metales pesados (E conomou, 2005; Xu, 2008).

El óxido de bismuto, $\mathrm{Bi}_{2} \mathrm{O}_{3}$, es uno de los óxidos más simples y muchos trabajos se han dedicado al estudio de sus propiedades fisicoquímicas. Con este material se han logrado obtener diferentes estructuras cristalográficas de dimensiones micrométricas y nanométricas llamadas polimorfos. Se caracteriza por ser un conductor iónico, es decir, los iones de oxígeno se mueven fácilmente a través de él, pues tiene una amplia banda de energía prohibida, un alto índice de refracción, una alta permitividad dieléctrica y una buena fotoconductividad. Estas propiedades lo convierten en un buen candidato para su uso en aplicaciones tales como las pilas de combustible, los sensores, los conductores iónicos, los materiales fotoeléctricos, los superconductores de alta temperatura y cerámicas funcionales. También es un componente importante en la manufactura de vidrios cerámicos transparentes, recubrimientos ópticos y cerámicos, entre otras aplicaciones (Bedoya, et al., 2012; Zhang, et al., 2011; Nowak-Wozny, et al., 2009; Wang, et al., 2011; Schuisky \& Harsta, 1996; Li, et al., 2007; Lee, et al., 2005).

En este trabajo se depositó bismuto y óxido de bismuto $\left(\mathrm{Bi}_{2} \mathrm{O}_{3}\right)$ en forma de películas delgadas mediante el sistema de magnetrón desbalanceado (unbalanced magnetron, UBM). Se estudiaron sus propiedades estructurales por medio de las técnicas de difracción de rayos X y microscopía óptica confocal; su composición se analizó con espectroscopia Auger y las propiedades ópticas con la técnica de espectroscopia ultravioleta visible (UV/Vis). Todo ello con el objeto de evaluar sus fases y estructura, así como su comportamiento óptico con respecto a las propiedades que dependen del método de crecimiento reportadas en la bibliografía.

\section{Materiales y métodos}

Las películas delgadas de bismuto y óxido de bismuto se fabricaron en un sistema de sputtering con magnetrón desbalanceado sobre sustratos de vidrio a temperatura ambiente, los cuales se limpiaron con acetona e isopropanol en ultrasonido antes de la deposición. Para generar la descarga se utilizó un blanco de bismuto (99,999 \% de pureza) con un diámetro de 10,1 cm. Para el crecimiento de los recubrimientos de bismuto se usó un ambiente gaseoso de argón de pureza ultra alta, y para el caso del óxido del bismuto, una mezcla de argón y oxígeno en una proporción de 20 a 80 . En la tabla 1 se resumen los parámetros para la deposición utilizados en la fabricación de las películas delgadas.
Tabla 1. Condiciones de deposición para la fabricación de las películas delgadas de bismuto y óxido de bismuto

\begin{tabular}{lcc}
\hline Parámetro & $\begin{array}{c}\text { Valor para } \\
\text { bismuto }\end{array}$ & $\begin{array}{c}\text { Valor para óxido de } \\
\text { bismuto }\end{array}$ \\
\hline Potencia & $40 \mathrm{~W}$ & $40 \mathrm{~W}$ \\
\hline Distancia sustrato blanco & $50 \mathrm{~mm}$ & $50 \mathrm{~mm}$ \\
\hline Presión base & $\sim 9 \times 10^{-4} \mathrm{~Pa}$ & $\sim 9 \times 10^{-4} \mathrm{~Pa}$ \\
\hline Presión trabajo & $\sim 0,5 \mathrm{~Pa}$ & $\sim 0,5 \mathrm{~Pa}$ \\
\hline Flujo de gas (argón) & $9 \mathrm{sccm}$ & $\begin{array}{c}(80 \% \text { argón }-20 \% \\
\text { oxígeno }) 9 \mathrm{sccm}\end{array}$ \\
\hline
\end{tabular}

El espesor de las películas se midió con un perfilómetro Dektak 150. Los tiempos de depósito se optimizaron para obtener un espesor final de las películas de aproximadamente $120 \mathrm{~nm}$.

Para la caracterización microestructural se hicieron medidas de difracción de rayos X (XRD) usando el equipo X-pert PRO PANalytical en el modo Bragg-Brentano, y con la línea monocromática $\mathrm{K} \alpha$ del cobre, cuya longitud de onda es de $1.540998 \AA$, una corriente de trabajo de $40 \mathrm{~mA}$, y una diferencia de potencial de $45 \mathrm{kV}$. La morfología de las películas se estudió con un microscopio óptico confocal Zeiss LSM 700 con poder de resolución máximo de 250 nm y una longitud de onda de $405 \mathrm{~nm}$. Para el análisis composicional de las muestras se usó el equipo de espectroscopia de electrones Auger (AES) de la marca Omicron Nanotechnolgy que cuenta con un espejo cilíndrico analizador (cylindrical mirror analyzer, CMA) de $3 \mathrm{KeV}$. Para la caracterización óptica se utilizó un espectrofotómetro Cary 5000 UV-VisNIR de alto rendimiento en la gama de 175 a $3.300 \mathrm{~nm}$, en condiciones de presión atmosférica normal y temperatura ambiente. Este sistema es controlado por el programa Cary WinUV basado en Windows. El rango de longitudes de onda fue de 500 a $1.800 \mathrm{~nm}$.

\section{Resultados y discusión}

\section{Caracterización microestructural}

En la figura 1 se muestra el espectro de difracción de rayos $\mathrm{X}$ obtenido para la película de bismuto. En general, las películas presentaron una estructura romboédrica ( $a=b \neq c, \alpha=\beta=$ $90^{\circ}, \gamma=120^{\circ}$ ), con una orientación mixta en varios planos cristalinos que se resumen en la tabla 2. Los parámetros de red presentaron valores de: $a=4,5839 \AA, b=4,5839 \AA$, $\mathrm{c}=12,1642 \AA$, menores a los reportados en el material en bulto (se utilizó como referencia la ficha del código $01-085$ - 1331), lo cual es un indicativo de tensiones residuales en la película. Esto también se corroboró con el cambio de las posiciones de los picos experimentales con respecto a los picos de difracción del patrón de referencia y puede explicarse por los efectos de re-nucleación y los defectos producto de la ausencia de condiciones de equilibrio en el crecimiento del recubrimiento (Albella, 2003). 


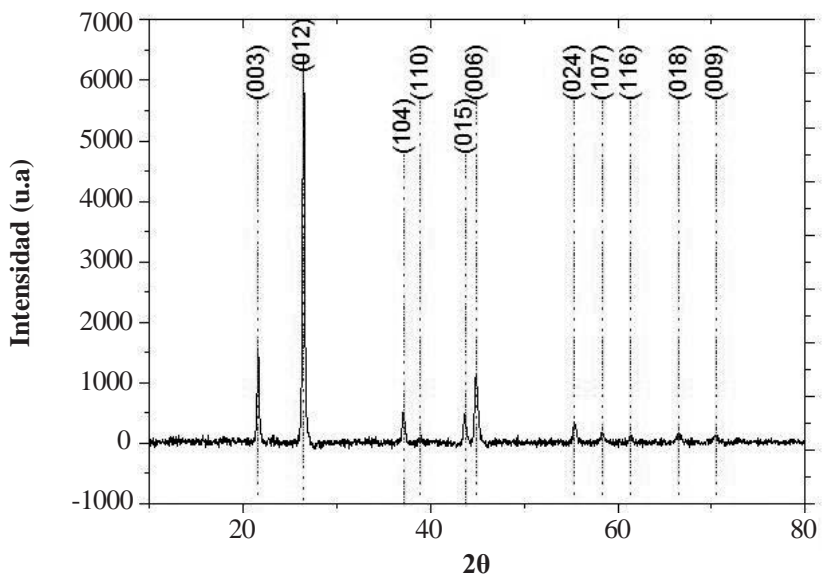

Figura 1. Patrón de difracción de rayos $\mathrm{X}$ para una muestra representativa de bismuto depositado por sputtering con magnetrón desbalanceado. Se presentan los índices de Miller correspondientes a los planos cristalinos asociados al material.

Tabla 2. Posiciones y planos de difracción en el bismuto obtenidos a partir del espectro XRD mostrado en la figura 1

\begin{tabular}{|c|c|c|c|}
\hline (hkl) & Experimental $2 \theta$ & Referencia $2 \theta$ & Incertidumbre (\%) \\
\hline 003 & 21,578 & 22,593 & 4,489001018 \\
\hline 012 & 26,417 & 27,266 & 3,110467249 \\
\hline 104 & 37,080 & 38,135 & 2,765963026 \\
\hline 110 & 38,967 & 39,737 & 1,935475753 \\
\hline 015 & 43,646 & 44,78 & 2,531040643 \\
\hline 006 & 44,855 & 46,13 & 2,762410579 \\
\hline 024 & 55,332 & 56,252 & 1,634253004 \\
\hline 107 & 58,303 & 59,658 & 2,269771028 \\
\hline 116 & 61,285 & 62,483 & 1,916681337 \\
\hline 018 & 66,418 & 67,836 & 2,090335515 \\
\hline 009 & 70,358 & 71,983 & 2,257060695 \\
\hline
\end{tabular}

\section{a.}

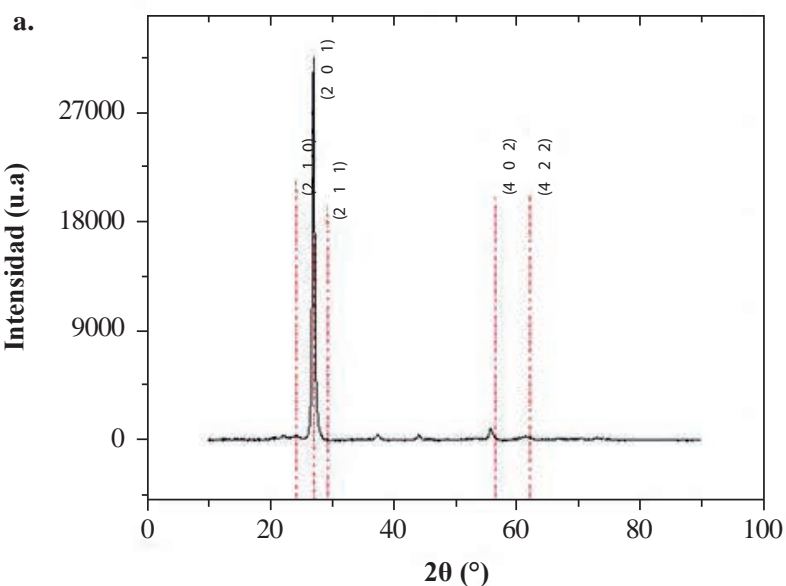

El patrón de difracción para la muestra de óxido de bismuto se muestra en la figura 2, y las direcciones y ángulos de difracción más importantes del espectro se resumen en la tabla 3. Se puede observar que las muestras de óxido de bismuto son policristalinas con una orientación mixta que corresponde a la fase de acuerdo con el PDF (Powder Diffraction File) de referencia $01-078$ - 1793, por lo que tiene una estructura tetragonal $(\alpha=\beta=\gamma=90)$. También se observó la presencia de bismuto sin oxidar con la misma estructura observada en la figura 1. Las constantes de red para el óxido de bismuto presente en estas muestras fueron de: $a=8,1747 \AA, b=8,1747 \AA, c=5,4110 \AA$.

Debido a la cercanía de los picos principales en el espectro de difracción de rayos X que presentan los distintos polimorfos del bismuto, las muestras de se analizaron por microscopía Raman con el objeto de confirmar su composición en fases. En la figura 2 b se muestra un espectro Raman para el óxido de bismuto crecido por sputtering con magnetrón desbalanceado.

Al igual que en el caso de lo encontrado en las muestras de bismuto, las películas delgadas de óxido de bismuto también presentaron tensiones residuales, lo cual se comprobó por el corrimiento de los picos en el patrón de difracción, es decir, por el cambio de las constantes de red.

\section{Microscopia láser confocal}

En la figura 3 a y b se muestra una micrografía con CLM de una muestra de bismuto y en la figura $3 \mathrm{c}$ y d la correspondiente a una muestra de óxido de bismuto, $\beta-\mathrm{Bi}_{2} \mathrm{O}_{3}$. Los aumentos de $500 \mathrm{X}$ y $1000 \mathrm{X}$ permitieron observar que las superficies eran homogéneas con presencia de capas o imperfecciones probablemente asociadas al proceso de deposición de estos tipos de recubrimientos. Con el análisis se obtuvo una rugosidad lineal media (Figura 3b) (calculada sobre la línea roja en la imagen de la figura $3 \mathrm{~b}$ ) de $R_{a L} \approx 0,033 \mu \mathrm{m}$, y una rugosidad superficial (calculada sobre toda el área mostrada

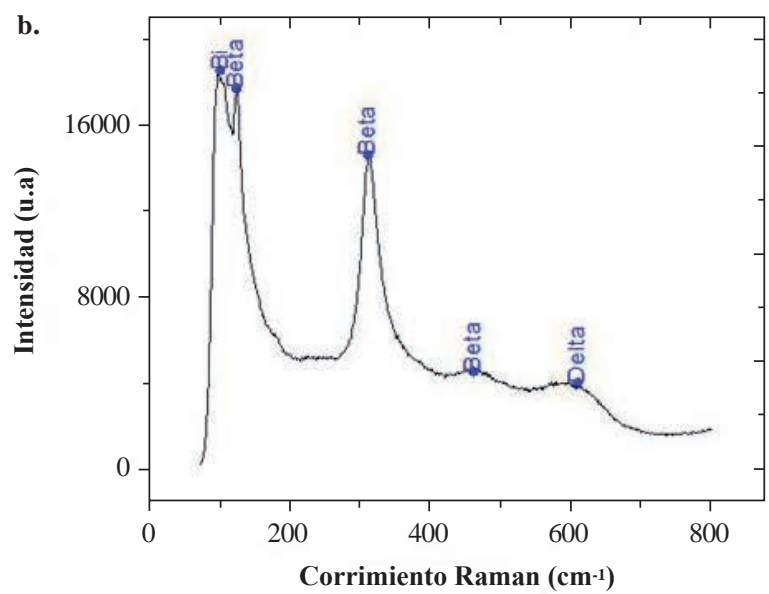

Figura 2. a. Patrón de difracción experimental de una muestra representativa de óxido de bismuto. Se muestran los picos correspondientes al óxido de bismuto en su fase $\beta$. b. Espectro Raman de una película delgada de óxido de bismuto depositado por sputtering con magnetrón desbalanceado. Se muestran los picos presentados etiquetados con sus respectivas fases. 
Tabla 3. Reporte de las posiciones y planos cristalinos para el óxido de bismuto obtenidos a partir del espectro XRD mostrado en la figura 3

\begin{tabular}{|c|c|c|c|c|c|}
\hline \multicolumn{3}{|c|}{ (hkl) } & Experimental 20 & Referencia $2 \theta$ & Incertidumbre (\%) \\
\hline 2 & 1 & 0 & 24,237 & 25,713 & 5,739898106 \\
\hline 2 & 0 & 1 & 26,998 & 27,945 & 3,386294507 \\
\hline 2 & 1 & 1 & 29,288 & 30,278 & 3,268709954 \\
\hline 4 & 0 & 2 & 56,498 & 57,752 & 2,171353373 \\
\hline 4 & 2 & 2 & 62,203 & 62,975 & 1,224454148 \\
\hline
\end{tabular}
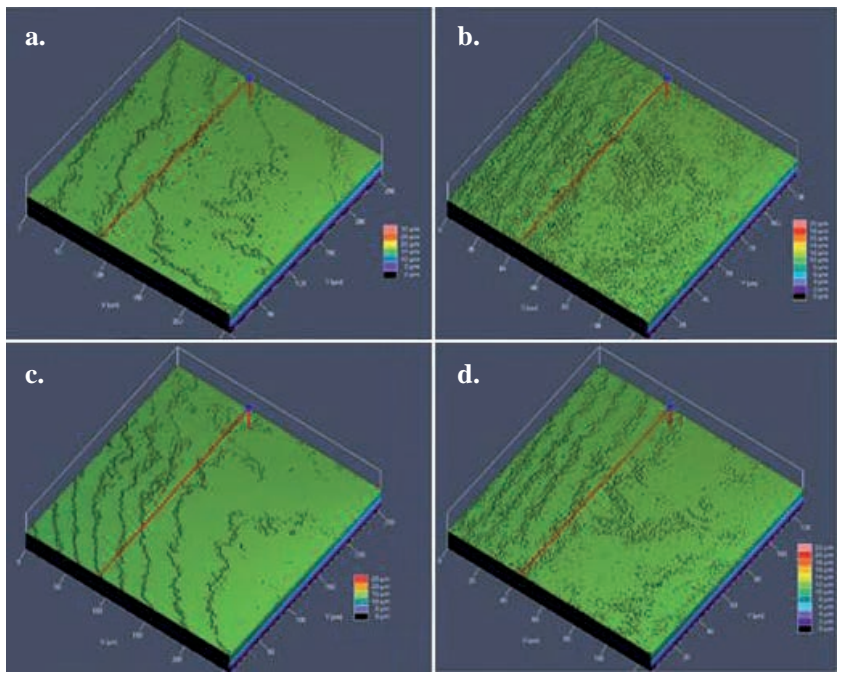

Figura 3. Microscopía confocal para una muestra de bismuto: a. $500 \mathrm{X}(250 \times 250 \mu \mathrm{m})$ y b. $1000 \mathrm{X}(120 \times 120 \mu \mathrm{m})$, y una muestra de óxido de bismuto $\beta$ : c. $500 \mathrm{X}(250 \times 250 \mu \mathrm{m})$ y d. $1000 \mathrm{X}$ $(120 \times 120 \mu m)$.

en la figura 3 b) de $120 \times 120 \mu \mathrm{m}$, de $R_{a A} \approx 0,096 \mu \mathrm{m}$. Las muestras de óxido de bismuto presentaron valores de rugosidad lineal y superficial (obtenidas a partir de la figura $3 \mathrm{~d}$ ) de: $R_{a L} \approx 0,028 \mu m$ y $R_{a A} \approx 0,092 \mu m$. Nótese que ambos depósitos mostraron una baja rugosidad, lo que se refleja en su apariencia física de brillo metálico tipo espejo; sin embargo, los recubrimientos de óxido de bismuto son, en general, menos rugosos que los de bismuto, lo que puede deberse a la disminución de la cantidad de partículas no fundidas en la superficies del óxido de bismuto, las cuales se forman durante el proceso de deposición debido al bajo punto de fusión del bismuto.

\section{Composición química}

En la figura 4a se muestra el espectro de electrones Auger de una muestra de bismuto. La energía cinética del electrón emitido fue igual a la energía electrónica Auger, dada por la posición del pico en el espectro, $E_{W X Y}$, resultante de la diferencia entre las energías de enlace de los niveles electrónicos involucrados en el proceso:

$$
E_{W X Y}=E_{X}-E_{W}-E_{Y}
$$

Para asignar a cada pico el elemento, así como su transición Auger, se usaron los datos tabulados en la literatura (Watts, 2003). Se puede observar que la mayoría de los picos corresponden al bismuto, a excepción de los correspondientes al silicio, el cual es del sustrato. Para obtener información del porcentaje en peso de cada elemento de la muestra por medio de la espectroscopia Auger, se hizo uso de la siguiente ecuación:

$$
x_{i}=\frac{y_{i} / s_{i}}{\sum_{\alpha} y_{\alpha} / s_{\alpha}} \times 100
$$

donde $x_{i}$ es la concentración en \% en peso del elemento i, $s_{i}$ es el factor de sensibilidad AES para el elemento i, en tanto que $y_{i}$ es la intensidad del pico. Los valores de la sensibilidad se encuentran tabulados (Watts, 2003). Así, la muestra resultó estar compuesta de $99 \%$ de bismuto y $1 \%$ de silicio.

Las posiciones de los picos, el elemento correspondiente, la transición que produce el electrón Auger y la energía de enlace obtenidos a partir de la figura 4a, se muestran en la tabla 4. Estas transiciones concuerdan con lo encontrado por
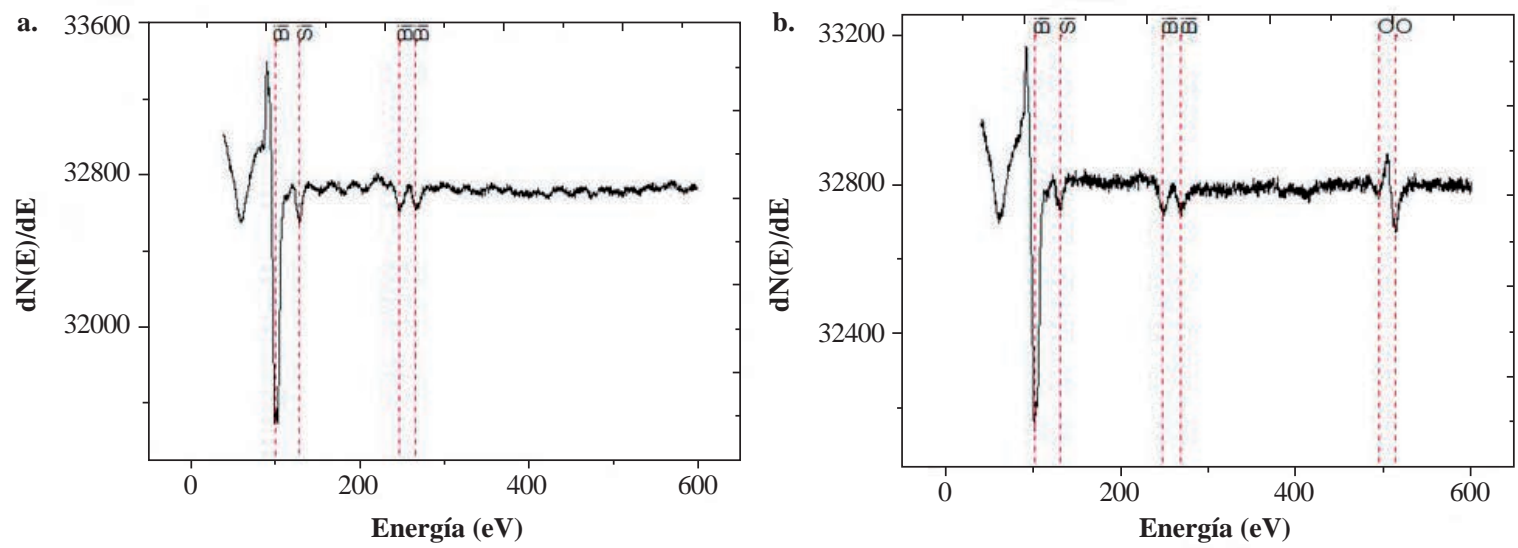

Figura 4. Espectro Auger para una muestra de: a. bismuto, b. óxido de bismuto. Se describen las señales de Bi (Bismuto), Si (Silicio) y O (Oxigeno). 
Tabla 4. Energías de enlace para las muestras de bismuto y óxido de bismuto según la figura 4

\begin{tabular}{lccc}
\hline \multicolumn{4}{c}{ Bismuto (figura 4 a) } \\
\hline Elemento & $\begin{array}{c}\text { Posición } \\
\text { del pico eV }\end{array}$ & Transición & $\begin{array}{c}\text { Energía } \\
\text { de enlace }\end{array}$ \\
\hline Bismuto & 101,74 & $\mathrm{~N}_{7} \mathrm{O}_{4} \mathrm{O}_{4}$ & 104 \\
\hline Silicio & 128,8 & $\mathrm{~L}_{1} \mathrm{M}_{1} \mathrm{M}_{1}$ & 133 \\
\hline Bismuto & 247,15 & $\mathrm{~N}_{5} \mathrm{O}_{3} \mathrm{O}_{3}$ & 254 \\
\hline Bismuto & 267,02 & $\mathrm{~N}_{4} \mathrm{O}_{3} \mathrm{O}_{3}$ & 278 \\
\hline \hline & Óxido de bismuto $\left(\mathbf{f i g u r a}_{2} \mathbf{~ b )}\right.$ & \\
\hline Bismuto & 101,2 & $\mathrm{~N}_{7} \mathrm{O}_{4} \mathrm{O}_{4}$ & 104 \\
\hline Silicio & 130,8 & $\mathrm{~L}_{1} \mathrm{M}_{1} \mathrm{M}_{1}$ & 133 \\
\hline Bismuto & 247,6 & $\mathrm{~N}_{5} \mathrm{O}_{3} \mathrm{O}_{3}$ & 254 \\
\hline Bismuto & 268,4 & $\mathrm{~N}_{4} \mathrm{O}_{3} \mathrm{O}_{3}$ & 278 \\
\hline Oxigeno & 493,7 & $\mathrm{KL}_{1} \mathrm{~L}_{1}$ & 484 \\
\hline Oxigeno & 513,9 & $\mathrm{KL}_{3} \mathrm{~L}_{3}$ & 518 \\
\hline
\end{tabular}

J ovalekic, et al., 2009). Como se mencionó anteriormente, la asignación de un elemento a cada pico se hace por medio de los datos tabulados (Watts, 2003).

En la figura $4 \mathrm{~b}$ se ve el espectro Auger para una muestra de óxido de bismuto. De acuerdo con los cálculos de la composición química, la muestra estaba compuesta de un $85 \%$ de bismuto, un $14 \%$ de oxígeno y un $1 \%$ de silicio proveniente del sustrato. La lista de posiciones de los picos, del elemento correspondiente, la transición y la energía de enlace se muestran en la tabla 4.

\section{Caracterización óptica}

El espectro de transmisión para la muestra de bismuto depositada sobre vidrio se muestra en la figura 5a. Esta medición se realizó en el rango de longitudes de onda de 500-1.800 nm. Debido a la apariencia física de las muestras, que resultaron tener un brillo metálico, se esperaba que la transmitancia fuera baja, al menos en el rango de longitudes de onda visibles. Esto se confirmó con los resultados de transmitancia; además, la absorbancia de la muestra también resultó ser baja, ya que se encontró dentro del rango de 1,1 a 1,5 \%.

Como puede observarse, la mayor parte de la radiación electromagnética incidente sobre la muestra es reflejada, una parte es transmitida y otra pequeña porción es absorbida. Así, cuando la transmisión disminuye, la reflexión y la absorción incrementan; también se puede observar que en el mínimo de transmisión se presenta un máximo en la reflexión y en la absorción.

Teniendo en cuenta la relación que hay entre el coeficiente de transmisión y el coeficiente de absorción óptica (B hattacharyya, et al., 2009; Swanepoel, 1983), se tiene que:

$$
T=\exp (-\alpha d)
$$

donde $T$ es la transmitancia, $\alpha$ es el coeficiente de absorción y $d$ es el espesor de la muestra. Sin embargo, primero hay que tener una buena aproximación del espesor real de la muestra, para lo cual se emplea el método propuesto por B hattacharyya, et al. (2009). En resumen, este método consiste en el cálculo de las constantes ópticas por medio del espectro de transmisión de una muestra mediante el modelo de Kramer-Kroning, con el fin de relacionar el índice de refracción con el coeficiente de absorción. Una vez obtenidos estos valores, se realiza un proceso de iteración por medio del cual se calcula teóricamente la transmisión en función de la longitud de onda. Al comparar estos valores teóricos de la transmisión con los resultados experimentales se obtiene una buena aproximación del valor del espesor de la muestra. Ahora bien, el valor de la banda de energía prohibida óptica se puede calcular con la siguiente ecuación (B hattacharyya, et al., 2009; J ayachandran, 1997):

$$
\alpha(h v)=\frac{\left(h v-E_{g}\right)^{n}}{h v}
$$

donde $h v$ es la energía de los fotones incidentes, $E_{g}$ es la banda de energía prohibida óptica entre la banda de valencia y la banda de conducción y $n$ es el exponente que caracteriza la transición electrónica, ya sea directa o indirecta. Así, $n$ es 1/2, 3/2, 2 y 3 para transiciones directas permitidas, directas prohibidas, indirectas permitidas e indirectas prohibidas, respectivamente. En este trabajo se analizó el caso de las transiciones directas permitidas, es decir, $n=1 / 2$.

Así, $E_{g}$ se obtiene graficando $(\alpha h v)^{2} v s . h v$ y extrapolando la sección lineal de esta gráfica hasta cero (Figura $5 b$ ). Como resultado, se obtuvo que para el bismuto:

$$
E_{g}=2,3 \pm 0,01 \mathrm{eV} \text {. }
$$

Este valor calculado es mucho menor al reportado por la literatura (Sandomirskii, 2003) para películas delgadas de bismuto depositadas por evaporación térmica con un espesor de aproximadamente $150 \mathrm{~nm}$, para las cuales $E_{g}=$ 4,4 eV (J ayachandran, 1997), y para películas de bismuto depositadas por sputtering con $E_{g}=4,0 \mathrm{eV}$ (Ortiz, 2012; E ISayed, 2006). Una posible explicación para este valor de banda de energía prohibida es la rugosidad del material: una superficie con una rugosidad alta genera una mayor cantidad de dispersión de fotones y, por lo tanto, a mayor rugosidad el valor de la energía de la banda de energía prohibida es menor, como también lo es la absorbancia (Ortiz, 2012). Por otro lado, un corrimiento en el valor puede deberse a vacancias reticulares, ya que las películas que contienen vacancias tendrán una mayor energía de banda de energía prohibida (Ortiz, 2012).

Por último, como resultado de la aplicación del método de B hattacharyya, et al. (Figura 5c), se obtuvo un espesor de la muestra de bismuto de $114,3 \pm 0,7 \mathrm{~nm}$, el cual difiere ligeramente del medido por perfilometría: $121,6 \pm 0,1 \mathrm{~nm}$. 

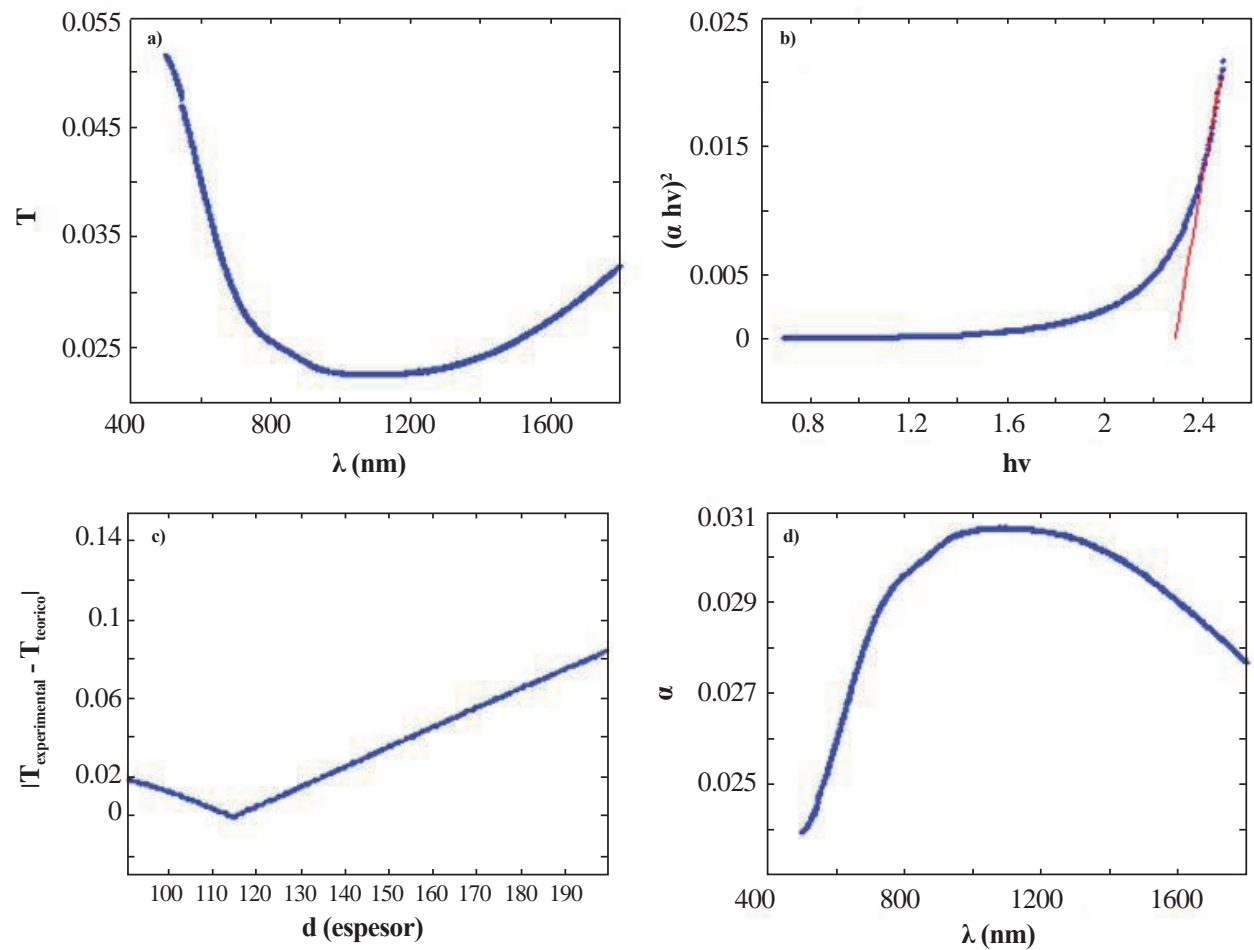

Figura 5. a. Transmitancia en función de la longitud de onda para el bismuto crecido por sputtering con magnetrón desbalanceado. b. Gráfica para el cálculo de la banda de energía prohibida en el recubrimiento de bismuto. c. Comparación entre la transmitancia teórica y la experimental. d. Coeficiente de absorción en función de la longitud de onda $\alpha(\lambda)$ para el bismuto depositado por sputtering con magnetrón desbalanceado.

Tabla 5. Porcentaje de error entre el espesor teórico (medido por perfilometría) y la energía de la banda de energía prohibida con los valores obtenidos experimentalmente

\begin{tabular}{lcc}
\hline & E spesor $(\mathbf{n m})$ & $\begin{array}{c}\text { Energía de banda de } \\
\text { energía prohibida }(\mathbf{e V})\end{array}$ \\
\hline Experimental & 114,3 & 2,3 \\
Teórico & 121,6 & 4,0 \\
\% de error & 6 & 42,5 \\
\hline
\end{tabular}

Con estos valores se determinó el coeficiente de absorción como función de la longitud de onda para el bismuto, tal como se muestra en la figura $5 \mathrm{~d}$. En la tabla 5 se resumen los resultados para el bismuto.

El espectro de transmisión para el óxido de bismuto crecido en este trabajo se muestra en la figura 6a. La transmitancia para el óxido de bismuto fue baja y menor que la presentada por la muestra de bismuto; además, la transmisión aumentó con el incremento de la longitud de onda, a diferencia del bismuto, en el que fue mayor en los extremos de longitudes de onda y tuvo un mínimo para las longitudes de onda media. Esto podría implicar una baja absorción o reflexión en longitudes de onda altas. Al igual que las muestras de bismuto, el óxido de bismuto $\beta$ resultó tener un brillo metálico, es decir, una alta reflexión, por lo que su absorción también fue baja, aunque mayor que la del bismuto.
$\mathrm{Al}$ analizar estos resultados se pudo calcular el valor de $E_{g}$ para el óxido de bismuto:

$$
E_{g}=2,27 \pm 0,02 \mathrm{eV} .
$$

Este es un valor ligeramente menor al obtenido para la muestra de bismuto. El valor de la banda de energía prohibida directa calculado en este trabajo fue muy cercano al reportado en la literatura (J ayachandran, 1997; He, et al., 2007; F an, et al., 2006; I ljinas, et al., 2010; L eontie, et al., 2005; Patil, et al., 2007; Patil, et al.; Yadav, et al., 2007; Sirota, et al., 2012), el cual es de aproximadamente 2,46 eV. Cabe aclarar que, según la técnica de preparación empleada, llegan a predominar ciertas fases del óxido de bismuto. La banda de energía prohibida directa de este material puede variar entre $2 \mathrm{eV}$ y 3,96 eV(G ujar, et al., 2006). Más específicamente, para la fase $\beta-\mathrm{Bi}_{2} \mathrm{O}_{3}$ el rango de variación de la banda de energía prohibida es de 2,47-3,4 eV (G ujar, et al., 2006). Con dicho valor es de esperarse que este material sea un poco mejor conductor que el bismuto puro.

Continuando con los cálculos, como puede verse en la figura $6 b$, el valor del espesor de la película de óxido de bismuto es de 129,6 \pm 0,7 nm, el cual difiere un poco del valor medido por perfilometría: $127,7 \pm 0,1 \mathrm{~nm}$.

El coeficiente de absorción para el óxido de bismuto como una función de la longitud de onda, se muestra en la figura 6c. La pequeña transmisión (Figura 6a) y la falta de interferencia en 

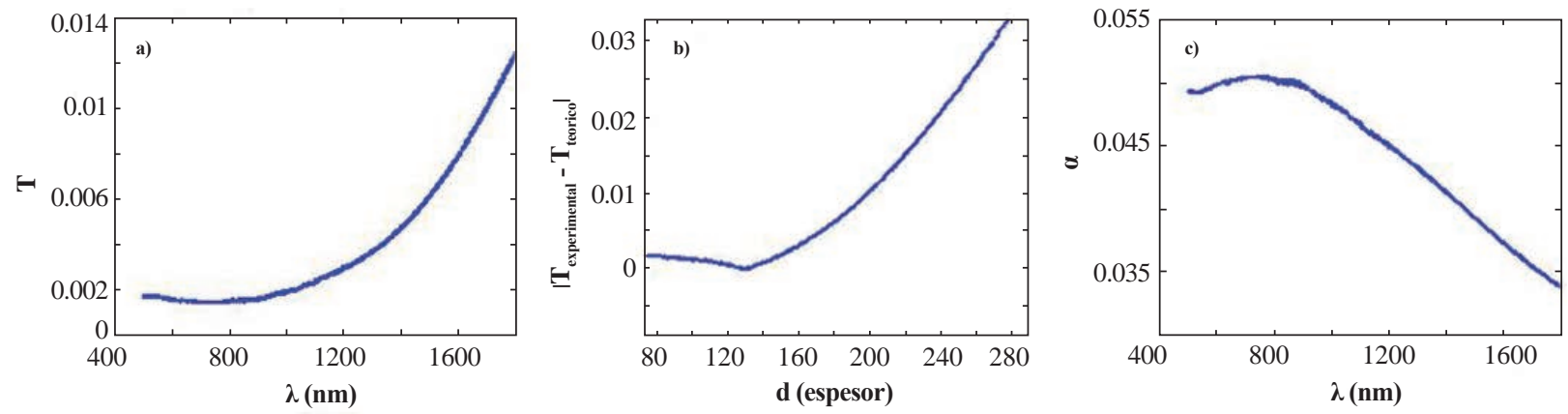

Figura 6. a. Transmisión en función de la longitud de onda para una muestra de óxido de bismuto depositada por sputtering con magnetrón desbalanceado. b. Curva utilizada para obtener el espesor de la película. c. Coeficiente de absorción en función de la longitud de onda para el óxido de bismuto depositado por sputtering con magnetrón desbalanceado.

Tabla 6. Comparación de los valores teóricos y los experimentales del espesor y la energía de la banda de energía prohibida del óxido de bismuto

\begin{tabular}{lcc}
\hline & E spesor $(\mathbf{n m})$ & Energía del band gap (eV) \\
\hline Experimental & 129,6 & 2,27 \\
Teórico & 127,7 & 2,46 \\
\% de error & 1,5 & 7,7 \\
\hline
\end{tabular}

el espectro puede ser consecuencia de que una gran fracción del material depositado sobre el sustrato de vidrio tiene una estructura amorfa, o puede relacionarse con un mayor grado de reflectancia o dispersión que de absorción (Iljinas, et al., 2010). Es claro que en el presente caso la conclusión tanto de los resultados de caracterización estructural por difracción de rayos $\mathrm{X}$ como de los obtenidos para las propiedades ópticas apunta a que la poca transparencia de las películas crecidas está más relacionada con una gran reflectancia que con la absorción. Esto concuerda con los resultados obtenidos por IIjinas, et al. (2010) para películas de óxido de bismuto depositadas por sputtering de corriente directa, a pesar de que en ese caso la composición en fase de la muestra es una combinación de las fases $\alpha$ y $\delta$.

En la tabla 6 se comparan los resultados obtenidos con los registrados como valores teóricos.

\section{Conclusiones}

Se prepararon películas delgadas de bismuto y óxido de bismuto mediante la técnica de sputtering con magnetrón desbalanceado. Con base en los análisis de difracción de rayos X, se concluyó que el bismuto presentaba la típica estructura romboédrica y los depósitos de $\mathrm{Bi}_{2} \mathrm{O}_{3}$ estaban compuestos principalmente de la fase tetragonal $\beta-\mathrm{Bi}_{2} \mathrm{O}_{3}$. Por medio de espectroscopia Raman también se observó la presencia de la fase $\delta-\mathrm{Bi}_{2} \mathrm{O}_{3}$. El análisis óptico mostró que tanto las muestras de bismuto como las de óxido de bismuto fueron altamente reflectivas, presentando una baja transmisión y un bajo índice de absorción. Sin embargo, las muestras de óxido de bismuto obtenidas por tratamiento térmico mostraron mayor transmisión y menor reflexión comparadas con las de bismuto. La película delgada de óxido de bismuto en su composición $\beta+$ $\delta$, en donde la fase beta es la dominante, presentó una energía de la banda de energía prohibida de 2,27 eV, menor que la del bismuto, la cual fue de 2,3 eV. Para el caso del depósito de bismuto, la energía prohibida resultó ser menor a lo reportado en la literatura. Este comportamiento probablemente se debe a la rugosidad de la muestra producto de la presencia de partículas no fundidas sobre su superficie. Otra explicación podría ser la poca presencia de vacancias de oxígeno.

\section{Conflicto de intereses}

Los autores declaran no tener ningún conflicto de intereses.

\section{Bibliografía}

Albella, J .M. 2003. Láminas delgadas y recubrimientos. Preparación, propiedades y aplicaciones. Editorial CSIC - CSIC Press. España. pp.101-123.

Bedoya, C. M., Pinzón, M.J., Orjuela, J.E.A., R estrepo, E., Olaya, J J . 2012. Physical-chemical properties of bismuth and bismuth oxides: Synthesis, characterization and applications. Dyna, 79 (176): 139-148.

Bhattacharyya, S.R., Gayen, R.N., Paul, R., Pal, A.K. 2009. Determination of optical constants of thin films from transmittance trace. Thin Solid Films. 517: 5530-5536. Disponible en: www.sciencedirect.com.

Boffoué, M. O., Lenoir, B., Scherrer, H., Dauscher, A. 1998. Pulsed laser deposition of bismuth in the presence of different ambient atmospheres. Thin Solid Films. 322: 132137. Disponible en: www.sciencedirect.com.

Depablos, O . L . 2013. Estabilidad estructural de películas delgadas de óxido de bismuto ( $\delta$-Bi_2 O_3). (Tesis de Maestría). Universidad Nacional Autónoma de México. México D.F.

Dresselhaus, M.S., Lin, Y.M., Rabin, O., J orio, A., Souza Filho, A.G ., Pimenta, M .A., Saito, R., Samsonidze, G., Dresselhaus, G . 2003. Nanowires and nanotubes. Materials Science and Engineering: C 23: 129-140. Disponible en: www.sciencedirect.com. 
E conomou, A. 2005. Bismuth - film electrodes: Recent developments and potentialities for electroanalysis. TrAC Trends in Analytical Chemistry. 24: 334-340. Disponible en: www. sciencedirect.com.

E I-Sayed, N.Z. 2006. Physical characteristics of thermally evaporated bismuth thin films. Vacuum. 80: 860-863. Disponible en: www.sciencedirect.com.

Fan, H.T., Pan, S.S., Teng, X.M., Ye, C ., Li, G.H ., Zhang, L .D. 2006. $\delta$-Bi2O3 thin films prepared by reactive sputtering: Fabrication and characterization. Thin Solid Films. 513: 142-147. Disponible en: www.sciencedirect.com.

G ujar, T.P, Shinde, V.R., L okhande, C.D. 2006. Spray pyrolysed bismuth oxide thin films and their characterization. Materials Research Bulletin. 41: 1558-1564. Disponible en: www.sciencedirect.com.

Hicks, L .D. \& Dresselhaus, M .S. 1993. Thermoelectric figure of merit of a one-dimensional conductor. Physical Review. B 47: 16631

Hofmann, P. 2006. The surfaces of bismuth: Structural and electronic properties. Progress in Surface Science. 81: 191-245. Disponible en: www.sciencedirect.com.

Iljinas, A., Burinskas, S., Dudonis, J . 2010. Synthesis of Bismuth Oxide Thin Films Deposited by Reactive Magnetron Sputtering. Proceedings of the VIII International Conference ION 2010, Kazimierz Dolny, Poland, June 14-17.

lyengar, A. S., Liang, D., Xuan, P.A., Abramson, A. R. 2012. Densification effects on the electrical behavior of uniaxially compacted bismuth nanowires. Acta Materialia. 60: 23692378. Disponible en: www.sciencedirect.com.

J ayachandran, K. 1997. Optical Studies in Bismuth, Antimony, Bismuth Oxide and Antimony Oxide Thin Films. (Thesis for awarding the degree of Doctor of Philosophy in Physics). Mahatma Gandhi University. School of Pure and Applied Physics.

J ovalekic', C ., Z duji'c, M., A tanasoska, Lj. 2009. Surface analysis of bismuth titanate by Auger and X-ray photoelectron spectroscopy. Journal of Alloys and Compounds. 469: 441444. Disponible en: www.sciencedirect.com.

L e, J .H ., O hara, S., Nagashima, T., H asegawa, T., Sugimoto, N., I garashi, K , K atoh, K., K ikuchi, K. 2005. Clock Recovery and Demultiplexing of High-Speed OTDM Signal Through Combined Use of Bismuth Oxide Nonlinear Fiber and Erbium-Doped Bismuth Oxide Fiber. IEEE Photonic Tech L. 17: 2658-2660. Disponible en: www.ieexplore.ieee.org.

L eontiea, L ., C aramanb, M ., Visinoiuc, A ., R usua, G .I . 2005. On the optical properties of bismuth oxide thin films prepared by pulsed laser deposition. Thin Solid Films. 473: 230-235. Disponible en: www.sciencedirect.com.

Li, L ., Yang, Y., Fang, X., K ong, M., Li, G ., Zhang, L . 2007. Diameter-dependent electrical transport properties of bismuth nanowire arrays. Communications. 141: 492-496. Disponible en: www.sciencedirect.com.

L in, Y.M ., Sun, X., Dresselhaus, M .S. 2000. Theoretical investigation of thermoelectric transport properties in cylindrical Bi nanowires, Physical Review. B 62: 4610.
L utskii, V.N. 1965. Features of optical absorption of metallic films in region where metal turns into a dielectric. Soviet Physics JETP Letters. 2: 245.

Nowak-Wozny, D., J aniczek, T., M ielcarek, W., G ajewski, J .B. 2009. Fractional electrical model for modified bismuth oxide. J. Electrostatics. 67: 18-21. Disponible en: www. sciencedirect.com

Ortiz, M. F.. 2012. Recubrimientos de bismuto depositados por la técnica sputtering D.C. pulsado. (Tesis de Maestría). Universidad Nacional de Colombia. Facultad de Ingeniería, Departamento de Mecánica y Mecatrónica. Bogotá D.C.

Patil, R.B., Yadav, J.B., Puri, R.K., Puri, V. 2007. Optical properties and adhesion of air oxidized vacuum evaporated bismuth thin films. Journal of Physics and Chemistry of Solids. 68: 665-669. Disponible en: www.sciencedirect.com.

Patil, R.B., Puri, R.K., Puri, V. 2007. Oxidation temperature dependent optical properties of bismuth oxide thin films: Effect of vapour chopping and air exposure. Applied Surface Science. 253: 8682-8688. Disponible en: www. sciencedirect.com.

Sandomirskii, V.B. 1967. Quantum size effect in a semimetal film. Soviet Physics JETP. 25: 101.

Schuisky, M. \& Harsta, A. 1996. Epitaxial growth of Bi2O2.33 by halide Cvd. Chem. Vap. Depos. 2 (6): 235-238.

Sirota, B., Reyes-C uellar, J ., Kohli, P., Wang, L., M cC arroll, M.E., A ouadi, S.M. 2012. Bismuth oxide photocatalytic nanostructures produced by magnetron sputtering deposition. Thin Solid Films. 520: 6118-6123. Disponible en: www.sciencedirect.com.

Swanepoel, R. 1983. Determination of the thickness and optical constants of amorphous silicon. J. Phps. E: Sci. Instrum. 16: 1214-1222. Disponible en: www.sciencedirect.com.

Wang, Y., Zhao, J, Wang. Z. 2011. A simple low-temperature fabrication of oblique prism-like bismuth oxide via a onestep aqueous process. Colloid Surface A. 377: 409-413. Disponible en: www.sciencedirect.com.

Watts, J . F. \& Wolstenholme, J . 2003. An Introduction to Surface Analysis by XPS and AES. Wiley. Chichester, Inglaterra. Cap. 1: 1-17; Cap. 3: 59-79.

Weidong, H., Wei, Q., Xiaohong, W., Hailong, N. 2007. Thin bismuth oxide films prepared through the sol-gel method. Materials Letters. 61: 4100-4102. Disponible en: www. sciencedirect.com.

$\mathbf{X} \mathbf{u}, \mathbf{H}, 2008$. A nafion-coated bismuth film electrode for the determination of heavy metals in vegetable using differential pulse anodic stripping voltametry: An alternative to mercury-based electrodes. Food Chemistry. 109: 834-839. Disponible en: www.sciencedirect.com.

Yang, Ch.. 2008. A Study of Electrical Properties in Bismuth Thin Films. Physics REU Summer. University of Florida. Gainesville, FL 32611. Disponible en: www.phys.ufl.edu

Zhang, L., Hashimoto, Y., Taishi, T., Nakamura, I., Ni, Q.-Q. 2011. Fabrication of flower-shaped Bi2O3 superstructure by a facile template-free process. Appl. Surf. Sci. 257: 6577-6582. Disponible en: www.sciencedirect.com. 